\title{
Asymptotic results for random walks in continuous time with alternating rates
}

\author{
Antonio Di Crescenzo* Claudio Macci ${ }^{\dagger} \quad$ Barbara Martinucci ${ }^{\ddagger}$
}

\begin{abstract}
We investigate some large deviation problems for a random walk in continuous time $\{N(t) ; t \geq$ $0\}$ with spatially inhomogeneous rates of alternating type. We first deal with the large deviation principle for the convergence of $N(t) / t$ to a suitable constant. Then, the case of moderate deviations is also discussed. Motivated by possible applications in chemical physics context, we finally obtain an asymptotic lower bound for level crossing probabilities both in the case of finite and infinite horizon.
\end{abstract}

AMS Subject Classification: 60F10; 60J27

Keywords: Large deviations, Moderate deviations, Probability generating function.

\section{Introduction}

Random walks in continuous time are largely employed in population dynamics, queueing theory, epidemiology, and many other areas of both theoretical and applied interest. In this paper we investigate certain features of skip-free random walks in continuous time over the whole set of integers, and having alternating rates, which extend some birth-death processes previously introduced in chemical context. Markov chains with alternating rates are useful in the study of chain molecular diffusion. We recall the paper [Stockmayer et al. (1971)], where a molecule is modeled as a freely-joined chain of two regularly alternating kinds of atoms, which have alternating jump rates. Moreover, a simple birth-death process with alternating rates has been studied in [Conolly et al. (1997)] as a model for an infinitely long chain of atoms joined by

\footnotetext{
*Dipartimento di Matematica, Università di Salerno, Via Giovanni Paolo II n. 132, 84084 Fisciano (SA), Italy. e-mail: adicrescenzo@unisa.it

${ }^{\dagger}$ Dipartimento di Matematica, Università di Roma Tor Vergata, Via della Ricerca Scientifica, 00133 Rome, Italy. e-mail: macci@mat.uniroma2.it

${ }^{\ddagger}$ Dipartimento di Matematica, Università di Salerno, Via Giovanni Paolo II n. 132, 84084 Fisciano (SA), Italy. e-mail: bmartinucci@unisa.it
} 
links which are subject to random alternating shocks. Recent results on the transient probabilities of such model, also in the presence of suitable reflecting or absorbing states, are provided in [Parthasarathy and Dharmaraja (1998)], [Tarabia (2009)], [Tarabia and El-Baz (2007)] and [Tarabia et al. (2009)]. An investigation on the two-periodic discrete-time random walk which is obtained by observing the previous process at its jump times has been performed in [Böhm and Hornik (2010)].

A random walk in continuous time characterized by alternating rates, in which the death rates are shifted with respect to those of [Conolly et al. (1997)], has been studied more recently in [Di Crescenzo et al. (2012)]. An extension of both such models is proposed and studied in the present paper. In Section 2 we describe the process and obtain the closed form of the probability generating function. The expressions of mean and variance are also provided. Section 3 is devoted to the study of large and moderate deviations for the proposed model. In particular, Section 3.2 deals with an asymptotic lower bound for level crossing probabilities of interest in chemical physics.

We remark that several results in the literature are concerning sample path large deviations for skip-free random walks in continuous time; see, for instance, the cases of scaled birth-death processes studied in [Chan (1998)] and in [Pakdaman et al. (2010)]. Sample path large deviations for general Markov processes are widely studied by Feng and Kurtz in [Feng and Kurtz (2006)]; a formulation of the main result in [Feng and Kurtz (2006)] for $d$-variate pure jump Markov processes is Theorem 5.1 in [Shwartz and Weiss (1995)], where the jump rates have to be regular functions defined on all $\mathbb{R}^{d}$. We also recall [Redig and Wang (2012)] where the connection of Feng-Kurtz theory with several examples of Gibbs-non-Gibbs transition of mean-field type is illustrated. The results in the present paper are concerning one dimensional random variables. The interest of this model relies on the fact that our proofs involve only the jump rates on $\mathbb{Z}$. Moreover, our results give more insight on the role of the parameters in the asymptotic behavior of the model (see e.g. Remark 3.1 on the limit value of the normalized process).

\section{The model and preliminary results}

Let $\{N(t) ; t \geq 0\}$ be a skip-free random walk in continuous time with state-space $\mathbb{Z}$, also known as 'bilateral birth-death process'. We denote its transition probabilities by

$$
p_{k, n}(t)=P\{N(t)=n \mid N(0)=k\}, \quad t \geq 0, \quad n \in \mathbb{Z},
$$

where $k \in \mathbb{Z}$ is the initial state. We assume that, for all $n \in \mathbb{Z}$, the upward transition rates are given by

$$
\lambda_{n}=\lim _{h \rightarrow 0} \frac{1}{h} P\{N(t+h)=n+1 \mid N(t)=n\}= \begin{cases}\lambda \eta+\mu(1-\eta), & n \text { even } \\ \mu \eta+\lambda(1-\eta), & n \text { odd }\end{cases}
$$


whereas the downward transition rates are

$$
\mu_{n}=\lim _{h \rightarrow 0} \frac{1}{h} P\{N(t+h)=n-1 \mid N(t)=n\}= \begin{cases}\lambda \theta+\mu(1-\theta), & n \text { even } \\ \mu \theta+\lambda(1-\theta), & n \text { odd }\end{cases}
$$

where $\lambda, \mu>0$, and $\theta, \eta \in[0,1]$.

According to the criteria developed in [Pruitt (1963)], from assumptions (1) and (2) it follows that both boundaries $-\infty$ and $+\infty$ are natural. We remark that the transition rates (1) and (2) have been defined as suitable extension of periodic rates treated in other models. Indeed, we notice that when $(\theta, \eta)=(0,1)$ the above model identifies with the model investigated by [Conolly et al. (1997)] and [Tarabia et al. (2009)], whereas when $(\theta, \eta)=(1,1)$ the rates $(1)$ and (2) refer to the bilateral birth-death process studied in [Di Crescenzo et al. (2012)]. Moreover, we notice that in the special case $\lambda=\mu$, process $\{N(t) ; t \geq 0\}$ identifies with the time-continuous nearest neighbor random walk with rate $\lambda$, studied in [Conolly (1971)].

Hereafter and throughout the paper we adopt the notation $\mathbb{E}_{k}[\cdot]=\mathbb{E}_{k}[\cdot \mid N(0)=k]$, and similarly $\operatorname{Var}_{k}[\cdot]=\operatorname{Var}[\cdot \mid N(0)=k]$.

Let us now obtain the probability generating function of $N(t)$, namely

$$
F_{k}(z, t):=\mathbb{E}_{k}\left[z^{N(t)}\right] \quad z>0, \quad t \geq 0,
$$

for any fixed initial state $k \in \mathbb{Z}$. To this purpose, for all $z, \alpha, \beta>0$ we define the following function:

$$
C(z ; \alpha, \beta):=[\eta \alpha+(1-\eta) \beta] z^{2}+(\alpha-\beta)(1-\theta-\eta) z+\theta \alpha+(1-\theta) \beta .
$$

Note that $C(z ; \alpha, \beta)>0$ for all $z \geq 0$. Indeed, if $(\alpha-\beta)(1-\theta-\eta) \geq 0$ then $C(0 ; \alpha, \beta)>0$ and $C(z ; \alpha, \beta)$ is increasing for $z>0$, whereas if $(\alpha-\beta)(1-\theta-\eta)<0$ then the discriminant of the quadratic polynomial in the right-hand-side of (4) is negative.

Proposition 2.1 For $z>0, t \geq 0$ and $k \in \mathbb{Z}$, the explicit expression of the probability generating function of $N(t)$ is:

$$
F_{k}(z, t)=e^{-(\lambda+\mu) t} z^{k}\left\{\cosh \left[t \frac{h(z)}{z}\right]+\frac{c_{k}(z)}{h(z)} \sinh \left[t \frac{h(z)}{z}\right]\right\},
$$

where

$$
c_{k}(z)= \begin{cases}C(z ; \lambda, \mu) & \text { if } k \text { is even } \\ C(z ; \mu, \lambda) & \text { if } k \text { is odd }\end{cases}
$$

with $C(z ; \cdot, \cdot)$ defined in $(4)$, and

$$
h(z)=\sqrt{\left(\mu z^{2}+\lambda\right)\left(\lambda z^{2}+\mu\right)+(\lambda-\mu)^{2}\left(1-z^{2}\right)\left[\theta(1-\theta)-z^{2} \eta(1-\eta)\right]} .
$$


Proof. For any $t \geq 0, n \in \mathbb{Z}$ and for any initial state $k \in \mathbb{Z}$, the transition probabilities of $N(t)$ satisfy the following system of differential-difference equations:

$$
\left\{\begin{aligned}
\frac{\mathrm{d}}{\mathrm{d} t} p_{k, 2 n}(t)= & {[\mu \eta+\lambda(1-\eta)] p_{k, 2 n-1}(t)+[\mu \theta+\lambda(1-\theta)] p_{k, 2 n+1}(t) } \\
- & {[\lambda \eta+\mu(1-\eta)+\lambda \theta+\mu(1-\theta)] p_{k, 2 n}(t) } \\
\frac{\mathrm{d}}{\mathrm{d} t} p_{k, 2 n+1}(t)= & {[\lambda \eta+\mu(1-\eta)] p_{k, 2 n}(t)+[\lambda \theta+\mu(1-\theta)] p_{k, 2 n+2}(t) } \\
- & {[\mu \eta+\lambda(1-\eta)+\mu \theta+\lambda(1-\theta)] p_{k, 2 n+1}(t) }
\end{aligned}\right.
$$

with initial condition $p_{k, n}(0)=\delta_{n, k}$, where $\delta_{n, k}$ is the Kronecker's delta. For $z>0$, let

$$
G_{k}(z, t):=\sum_{j=-\infty}^{+\infty} z^{2 j} p_{k, 2 j}(t), \quad H_{k}(z, t):=\sum_{j=-\infty}^{+\infty} z^{2 j+1} p_{k, 2 j+1}(t),
$$

be the probability generating functions of the sets of even and odd states of $N(t)$, respectively. From system (8) it follows that the generating functions (9) satisfy the differential system

$$
\frac{\partial}{\partial t}\left[\begin{array}{c}
G_{k}(z, t) \\
H_{k}(z, t)
\end{array}\right]=A \cdot\left[\begin{array}{c}
G_{k}(z, t) \\
H_{k}(z, t)
\end{array}\right]
$$

where

$$
A:=\left[\begin{array}{cc}
-[\lambda \eta+\mu(1-\eta)+\lambda \theta+\mu(1-\theta)] & \frac{\mu \theta+\lambda(1-\theta)+z^{2}[\mu \eta+\lambda(1-\eta)]}{z} \\
\frac{\lambda \theta+\mu(1-\theta)+z^{2}[\lambda \eta+\mu(1-\eta)]}{z} & -[\mu \eta+\lambda(1-\eta)+\mu \theta+\lambda(1-\theta)]
\end{array}\right]
$$

with initial conditions

$$
G_{k}(z, 0)=\left\{\begin{array}{ll}
z^{k}, & k \text { even } \\
0, & k \text { odd },
\end{array} \quad H_{k}(z, 0)= \begin{cases}0, & k \text { even } \\
z^{k}, & k \text { odd } .\end{cases}\right.
$$

Hence, by solving system (10) and recalling relation $F_{k}(z, t)=G_{k}(z, t)+H_{k}(z, t)$ we immediately come to Eq. (5).

We are now able to obtain the mean of $N(t)$ conditional on the initial state. This is expressed in terms of

$$
M(\alpha, \beta):=\frac{(\alpha-\beta)(\eta-\theta)[(\alpha-\beta)(\eta+\theta-1)+(\alpha+\beta)]}{2(\alpha+\beta)^{2}} .
$$

Proposition 2.2 For any initial state $k \in \mathbb{Z}$ and for all $t \geq 0$ the explicit expression of the mean of $N(t)$ is:

$$
\mathbb{E}_{k}[N(t)]=k+m_{k}\left(1-e^{-2(\lambda+\mu) t}\right)+\frac{(\lambda-\mu)^{2}[\eta(1-\eta)-\theta(1-\theta)]}{\lambda+\mu} t
$$

where

$$
m_{k}= \begin{cases}M(\lambda, \mu) & \text { if } k \text { is even } \\ M(\mu, \lambda) & \text { if } k \text { is odd }\end{cases}
$$

with $M(\cdot, \cdot)$ defined in (11). 
Remark 2.1 From Proposition 2.2 we have

$$
\lim _{t \rightarrow+\infty} \frac{1}{t} \mathbb{E}_{k}[N(t)]=\frac{(\lambda-\mu)^{2}[\eta(1-\eta)-\theta(1-\theta)]}{\lambda+\mu}=: \ell_{1},
$$

where $\ell_{1}=0$ if $\lambda=\mu$, or $\theta=\eta$, or $\theta=1-\eta$. We also note that, if $\lambda \neq \mu$, we have:

- $\ell_{1}>0$ if and only if $\eta(1-\eta)>\theta(1-\theta)$, i.e. if and only if $\left|\eta-\frac{1}{2}\right|<\left|\theta-\frac{1}{2}\right|$;

- $\ell_{1}<0$ if and only if $\eta(1-\eta)<\theta(1-\theta)$, i.e. if and only if $\left|\eta-\frac{1}{2}\right|>\left|\theta-\frac{1}{2}\right|$.

Let us now obtain the variance of $N(t)$ conditional on the initial state.

Proposition 2.3 For all $t \geq 0$, if $k$ is even the conditional variance of $N(t)$ is:

$$
\operatorname{Var}_{k}[N(t)]=a_{1} t+a_{2} t\left(1-2 e^{-2(\lambda+\mu) t}\right)-a_{3}\left(1-e^{-2(\lambda+\mu) t}\right)^{2}-a_{4}\left(1-e^{-2(\lambda+\mu) t}\right),
$$

where

$$
\begin{aligned}
a_{1} & =\frac{1}{(\lambda+\mu)^{3}}\left\{4(1-\eta) \eta(\lambda-\mu)^{2}(\lambda+\mu)^{2}+4 \lambda \mu(\lambda+\mu)^{2}+(\lambda-\mu)^{2}(\eta-\theta)(-1+\eta+\theta)\right. \\
& \left.\times\left[2(\lambda+\mu)^{2}-2(\lambda-\mu)^{2}(\eta-\theta)(-1+\eta+\theta)-(\lambda-\mu)(\lambda+\mu)(\eta-\theta)\right]\right\}, \\
a_{2} & =\frac{1}{(\lambda+\mu)^{3}}(\lambda-\mu)^{3}(\eta-\theta)^{2}(-1+\eta+\theta)[(\lambda-\mu)(-1+\eta+\theta)+\lambda+\mu], \\
a_{3} & =\frac{(\lambda-\mu)^{2}(\eta-\theta)^{2}[(\lambda-\mu)(-1+\eta+\theta)+\lambda+\mu]^{2}}{4(\lambda+\mu)^{4}}, \\
a_{4} & =\frac{(\lambda-\mu)}{2(\lambda+\mu)^{4}}\left\{( 2 \eta - 1 ) ( \lambda - \mu ) ( 2 \theta - 1 ) \left[2(\lambda-\mu)^{2}(-1+\eta+\theta)^{2}\right.\right. \\
& \left.+[(-1+\eta+\theta)(\lambda-\mu)+\lambda+\mu]^{2}\right]-3(\lambda-\mu)^{3}(-1+\eta+\theta)^{4}-(\lambda+\mu)(-1+\eta+\theta) \\
& \left.\times\left[(\lambda-\mu)^{2}(-1+\eta+\theta)^{2}+[(-1+\eta+\theta)(\lambda-\mu)+\lambda+\mu]^{2}\right]\right\} .
\end{aligned}
$$

If $k$ is odd, $\operatorname{Var}_{k}[N(t)]$ is obtained by Eq. (13) by interchanging $\lambda$ with $\mu$.

The proof of Propositions 2.2 and 2.3 is omitted, since it follows from Eq. (5) via straightforward calculations.

Remark 2.2 From Proposition 2.3 we have

$$
\begin{aligned}
\lim _{t \rightarrow+\infty} \frac{1}{t} \operatorname{Var}_{k}[N(t)]= & \frac{1}{(\lambda+\mu)^{3}}\left\{-(\eta-\theta)^{2}(1-\eta-\theta)^{2}(\lambda-\mu)^{4}-2(\eta-\theta)(1-\eta-\theta)\left(\lambda^{2}-\mu^{2}\right)^{2}\right. \\
& \left.+4 \eta(1-\eta)\left(\lambda^{2}-\mu^{2}\right)^{2}+4 \lambda \mu(\lambda+\mu)^{2}\right\}:=\ell_{2} .
\end{aligned}
$$

Note that $\ell_{2}>0$ for all $\lambda, \mu>0,0 \leq \eta \leq 1$ and $0 \leq \theta \leq 1$. Clearly, it is $\ell_{2}=a_{1}+a_{2}$. 
Finally we focus on an interesting property of the process $\{N(t) ; t \geq 0\}$, which is related to its asymptotic behavior as $\lambda$ grows larger. We recall that a family of random variables $U^{(\lambda)}$ exhibits cut-off behavior at mean times if (see, for instance, Definition 1 of [Barrera et al. (2009)])

$$
\lim _{\lambda \rightarrow+\infty} P\left(U^{(\lambda)}>c \mathbb{E}\left[U^{(\lambda)}\right]\right)= \begin{cases}1 & \text { for } c<1, \\ 0 & \text { for } c>1 .\end{cases}
$$

Proposition 2.4 Let $\eta, \theta \in(0,1)$. If $\eta \neq \theta$ and $\eta \neq 1-\theta$, then process $\{N(t) ; t \geq 0\}$ exhibits cut-off behavior at mean times with respect to $\lambda$.

Proof. A sufficient condition for $\{N(t) ; t \geq 0\}$ to exhibit cut-off behavior is given by (see Proposition 1 of [Barrera et al. (2009)])

$$
\lim _{\lambda \rightarrow+\infty} \frac{\operatorname{Var}_{k}[N(t)]}{\left(\mathbb{E}_{k}[N(t)]\right)^{2}}=0
$$

Since, from Eqs. (12) and (13), for $t>0$ we have

$$
\lim _{\lambda \rightarrow+\infty} \frac{1}{\lambda} \mathbb{E}_{k}[N(t)]=(\eta-\theta)(1-\eta-\theta) t,
$$

and

$$
\lim _{\lambda \rightarrow+\infty} \frac{1}{\lambda} \operatorname{Var}_{k}[N(t)]=\left[4(1-\eta) \eta-2(\eta-\theta)(1-\eta-\theta)-(\eta-\theta)^{2}(1-\eta-\theta)^{2}\right] t,
$$

the proof thus immediately follows.

\section{$3 \quad$ Large and moderate deviations}

We start by recalling some basic definitions on large deviations (see e.g. [Dembo and Zeitouni (1998)], pages 4-5). A family of probability measures $\left\{\pi_{t}: t>0\right\}$ on a topological space $z$ satisfies the large deviation principle (LDP for short) with rate function $I$ and speed function $v_{t}$ if: $\lim _{t \rightarrow+\infty} v_{t}=+\infty, I: z \rightarrow[0,+\infty]$ is lower semicontinuous,

$$
\liminf _{t \rightarrow+\infty} \frac{1}{v_{t}} \log \pi_{t}(O) \geq-\inf _{z \in O} I(z)
$$

for all open sets $O$, and

$$
\limsup _{t \rightarrow+\infty} \frac{1}{v_{t}} \log \pi_{t}(C) \leq-\inf _{z \in C} I(z)
$$

for all closed sets $C$. A rate function is said to be good if all its level sets $\{\{z \in z: I(z) \leq \eta\}$ : $\eta \geq 0\}$ are compact.

We prove two LDPs with $z=\mathbb{R}$. In both cases the proof consists of an application of the Gärtner Ellis Theorem recalled in the Appendix, where we take suitable limits in terms of the generating function $F_{k}$ given in Proposition 2.1. We also present a lower bound for level crossing probabilities in the final part of this section. 


\subsection{Large deviation principles}

We start with the LDP for the convergence of $N(t) / t$ to some constant as $t \rightarrow+\infty$, with $v_{t}=t$. Throughout this section we consider the function $\Lambda: \mathbb{R} \rightarrow \mathbb{R}$ defined by

$$
\Lambda(\gamma):=\frac{h\left(e^{\gamma}\right)}{e^{\gamma}}-(\lambda+\mu)
$$

where $h(\cdot)$ is defined in $(7)$.

Proposition 3.1 For all $k \in \mathbb{Z},\left\{P\left(\frac{N(t)}{t} \in \cdot \mid N(0)=k\right): t>0\right\}$ satisfies the LDP with speed function $v_{t}=t$ and good rate function $\Lambda^{*}$ defined by $\Lambda^{*}(z):=\sup _{\gamma \in \mathbb{R}}\{\gamma z-\Lambda(\gamma)\}$.

Proof. In order to apply the Gärtner Ellis Theorem we have to check that

$$
\lim _{t \rightarrow+\infty} \frac{1}{t} \log F_{k}\left(e^{\gamma}, t\right)=\Lambda(\gamma)
$$

for all $\gamma \in \mathbb{R}$, where $F_{k}$ is defined in (3). Firstly we remark that, from Eqs. (6) and (7), and from the positivity of $C(z ; \alpha, \beta)$, we have $h(z), c_{k}(z), \cosh \left(t \frac{h(z)}{z}\right), \sinh \left(t \frac{h(z)}{z}\right)>0$ for all $z, t>0$. Hence, since

$$
\cosh \left(t \frac{h\left(e^{\gamma}\right)}{e^{\gamma}}\right)+\frac{c_{k}\left(e^{\gamma}\right)}{h\left(e^{\gamma}\right)} \sinh \left(t \frac{h\left(e^{\gamma}\right)}{e^{\gamma}}\right) \geq \cosh \left(t \frac{h\left(e^{\gamma}\right)}{e^{\gamma}}\right)
$$

we have

$$
\liminf _{t \rightarrow+\infty} \frac{1}{t} \log \left[\cosh \left(t \frac{h\left(e^{\gamma}\right)}{e^{\gamma}}\right)+\frac{c_{k}\left(e^{\gamma}\right)}{h\left(e^{\gamma}\right)} \sinh \left(t \frac{h\left(e^{\gamma}\right)}{e^{\gamma}}\right)\right] \geq \frac{h\left(e^{\gamma}\right)}{e^{\gamma}} .
$$

Moreover, by taking into account that

$$
\limsup _{t \rightarrow+\infty} \frac{1}{t} \log \left[\cosh \left(t \frac{h\left(e^{\gamma}\right)}{e^{\gamma}}\right)\right]=\frac{h\left(e^{\gamma}\right)}{e^{\gamma}}
$$

and

$$
\limsup _{t \rightarrow+\infty} \frac{1}{t} \log \left[\frac{c_{k}\left(e^{\gamma}\right)}{h\left(e^{\gamma}\right)} \sinh \left(t \frac{h\left(e^{\gamma}\right)}{e^{\gamma}}\right)\right]=\frac{h\left(e^{\gamma}\right)}{e^{\gamma}}
$$

and by a simple application of Lemma 1.2.15 in [Dembo and Zeitouni (1998)], we get

$$
\limsup _{t \rightarrow+\infty} \frac{1}{t} \log \left[\cosh \left(t \frac{h\left(e^{\gamma}\right)}{e^{\gamma}}\right)+\frac{c_{k}\left(e^{\gamma}\right)}{h\left(e^{\gamma}\right)} \sinh \left(t \frac{h\left(e^{\gamma}\right)}{e^{\gamma}}\right)\right]=\frac{h\left(e^{\gamma}\right)}{e^{\gamma}} .
$$

In conclusion (16) and (17) yield

$$
\lim _{t \rightarrow+\infty} \frac{1}{t} \log \left[\cosh \left(t \frac{h\left(e^{\gamma}\right)}{e^{\gamma}}\right)+\frac{c_{k}\left(e^{\gamma}\right)}{h\left(e^{\gamma}\right)} \sinh \left(t \frac{h\left(e^{\gamma}\right)}{e^{\gamma}}\right)\right]=\frac{h\left(e^{\gamma}\right)}{e^{\gamma}},
$$

and due to (5) and (14) it is easy to check that this limit is equivalent to (15).

Remark 3.1 (On the limit value $\Lambda^{\prime}(0)$ ) A well-known consequence of the LDP in Proposition 3.1 is that $N(t) / t$ converges to $\Lambda^{\prime}(0)$ as $t \rightarrow+\infty$, where

$$
\Lambda^{\prime}(0)=\frac{(\lambda-\mu)^{2}[\eta(1-\eta)-\theta(1-\theta)]}{\lambda+\mu} .
$$


In particular, by taking into account Remark 2.1 we note that

$$
\Lambda^{\prime}(0)=\lim _{t \rightarrow+\infty} \frac{\mathbb{E}_{k}[N(t)]}{t}
$$

for all $k \in \mathbb{Z}$.

Remark 3.2 (On the expression of the rate function) It is not hard to see that, for all $z \in$ $\mathbb{R}$, there exists a (unique) $\gamma_{z} \in \mathbb{R}$ such that $\Lambda^{\prime}\left(\gamma_{z}\right)=z$, and therefore $\Lambda^{*}(z)=z \gamma_{z}-\Lambda\left(\gamma_{z}\right)$. In general an explicit expression for $\gamma_{z}$ is not feasible, and thus we cannot give an explicit form for $\Lambda^{*}(z)$. On the contrary, as we shall see, we can provide a closed-form expression for $\Lambda^{*}(z)$ when $\mu=\lambda$ (and for any choice of $\theta$ and $\eta$ ). In this case, we have

$$
\Lambda(\gamma)=\frac{\lambda\left(e^{2 \gamma}+1\right)}{e^{\gamma}}-2 \lambda=\lambda\left(e^{\gamma}-e^{-\gamma}\right)-2 \lambda \quad(\text { for all } \gamma \in \mathbb{R})
$$

so that, for all $z \in \mathbb{R}$, the equation $\Lambda^{\prime}(\gamma)=z$ admits solution $\gamma=\gamma_{z}=\log \frac{z+\sqrt{z^{2}+4 \lambda^{2}}}{2 \lambda}$. Hence, by substituting the value $\gamma_{z}$ in $\Lambda^{*}(z)=z \gamma_{z}-\Lambda\left(\gamma_{z}\right)$, we obtain

$$
\Lambda^{*}(z)=z \log \frac{z+\sqrt{z^{2}+4 \lambda^{2}}}{2 \lambda}-\sqrt{z^{2}+4 \lambda^{2}}+2 \lambda .
$$

Rate functions having this kind of expression appear in several references; for instance a slight modification is given in eq. (13) in [Ermolaev and Külske (2010)] for the "non-interacting dynamics' version of a model of interest statistical physics. We also remark that, when $\mu=\lambda$ (and for any choice of $\theta$ and $\eta$ ), we have a difference between two independent Poisson processes with intensity $\lambda$; so we can refer to the sample path large deviations for Lévy processes and, for any fixed $T \in(0, \infty)$, we have the $L D P$ in $[0, T]$ with speed function $v_{t}=t$ and good rate function $I_{[0, T]}$ defined by

$$
I_{[0, T]}(f)= \begin{cases}\int_{0}^{T} \Lambda^{*}(\dot{f}(s)) d s & \text { if } f \text { is absolutely continuous and } f(0)=0 \\ \infty & \text { otherwise }\end{cases}
$$

(see e.g. Theorem 1.2 in [de Acosta (1994)]). If we combine this result with suitable applications of the contraction principle (see e.g. Theorem 4.2.1 in [Dembo and Zeitouni (1998)]) we can obtain LDPs for finite dimensional random variables which can be considered as continuous-time versions of the Cramér Theorem (see e.g. Section 2.2 in [Dembo and Zeitouni (1998)]).

Now we study the moderate deviations. Roughly speaking we prove a class of LDPs with the same quadratic rate function which (uniquely) vanishes at the origin. In this way we typically fill the gap between the convergence of a family of random variables to 0 and an asymptotic normality result (as $t \rightarrow+\infty)$.

To better explain this concept, we recall the basic result on moderate deviations for the empirical means $\left\{\frac{X_{1}+\cdots+X_{n}}{n}: n \geq 1\right\}$ of i.i.d. centered and $\mathbb{R}^{d}$-valued random variables $\left\{X_{n}: n \geq\right.$ 
1) which have (common) finite moment generating function in a neighborhood of the origin and invertible covariance matrix $C$ (see e.g. Theorem 3.7.1 in [Dembo and Zeitouni (1998)]). In such a case we have the LDP for $\left\{\sqrt{n a_{n}} \frac{X_{1}+\cdots+X_{n}}{n}: n \geq 1\right\}$ for $\left\{a_{n}: n \geq 1\right\}$ such that $a_{n} \rightarrow 0$ and $n a_{n} \rightarrow+\infty($ as $n \rightarrow+\infty)$ with speed function $v_{n}=\frac{1}{a_{n}}$ and good rate function $J(z)=\frac{1}{2}\left\langle z, C^{-1} z\right\rangle$, where $\langle\cdot, \cdot\rangle$ is the inner product in $\mathbb{R}^{d}$. Thus we fill the gap between the law of large numbers (case $a_{n}=\frac{1}{n}$ ) and the central limit theorem (case $a_{n}=1$ ).

For the model studied in this paper we have to consider the convergence of $N(t) / t$ to $\Lambda^{\prime}(0)$ stated in Remark 3.1 (after a centering of the random variables), and the weak convergence of $\frac{N(t)-\mathbb{E}_{k}[N(t)]}{\sqrt{t}}$ to the centered Normal distribution with variance $\Lambda^{\prime \prime}(0)$ yielded by

$$
\lim _{t \rightarrow+\infty} \log \mathbb{E}_{k}\left[e^{\gamma \frac{N(t)-\mathbb{E}_{k}[N(t)]}{\sqrt{t}}}\right]=\frac{1}{2} \Lambda^{\prime \prime}(0) \gamma^{2}(\text { for all } \gamma \in \mathbb{R})
$$

proved in the Appendix (see e.g. Lemma 1(b) in [Cox and Griffeath (1984)] and, in particular, the remark after the statement of that lemma). We remark that this kind of problem, i.e. the weak convergence to a centered Normal distribution whose variance is determined by a LDP obtained by the Gärtner Ellis Theorem, has been already investigated in the literature; here we recall $[$ Bryc (1993)] and the references cited therein.

Proposition 3.2 Let $\left\{a_{t}: t>0\right\}$ be such that $a_{t} \rightarrow 0$ and $t a_{t} \rightarrow+\infty$ (as $\left.t \rightarrow+\infty\right)$. Then, for all $k \in \mathbb{Z},\left\{P\left(\sqrt{t a_{t}} \frac{N(t)-\mathbb{E}_{k}[N(t)]}{t} \in \cdot \mid N(0)=k\right): t>0\right\}$ satisfies the LDP with speed function $v_{t}=\frac{1}{a_{t}}$ and good rate function $J$ defined by $J(z):=\frac{z^{2}}{2 \Lambda^{\prime \prime}(0)}$.

Proof. In order to apply the Gärtner Ellis Theorem, by taking into account the equality $J(z)=$ $\sup _{\gamma \in \mathbb{R}}\left\{\gamma z-\frac{1}{2} \Lambda^{\prime \prime}(0) \gamma^{2}\right\}$ for all $z \in \mathbb{R}$, we have to check that

$$
\lim _{t \rightarrow+\infty} a_{t} \log \mathbb{E}_{k}\left[\exp \left(\frac{\gamma}{a_{t}} \sqrt{t a_{t}} \frac{N(t)-\mathbb{E}_{k}[N(t)]}{t}\right)\right]=\frac{1}{2} \Lambda^{\prime \prime}(0) \gamma^{2}
$$

for all $\gamma \in \mathbb{R}$. Firstly we note that, recalling (3), the limit (20) is equivalent to

$$
\lim _{t \rightarrow+\infty} a_{t} \log \left(F_{k}\left(e^{\gamma / \sqrt{t a_{t}}}, t\right) e^{-\gamma \mathbb{E}_{k}[N(t)] / \sqrt{t a_{t}}}\right)=\frac{1}{2} \Lambda^{\prime \prime}(0) \gamma^{2} .
$$

Moreover from (5) we have

$$
\begin{aligned}
F_{k}\left(e^{\gamma / \sqrt{t a_{t}}}, t\right) e^{-\gamma \mathbb{E}_{k}[N(t)] / \sqrt{t a_{t}}}=e^{-(\lambda+\mu) t} e^{\gamma\left(k-\mathbb{E}_{k}[N(t)]\right) / \sqrt{t a_{t}}} \\
\cdot\left[\cosh \left(t \frac{h\left(e^{\gamma / \sqrt{t a_{t}}}\right)}{e^{\gamma / \sqrt{t a_{t}}}}\right)+\frac{c_{k}\left(e^{\gamma / \sqrt{t a_{t}}}\right)}{h\left(e^{\gamma / \sqrt{t a_{t}}}\right)} \sinh \left(t \frac{h\left(e^{\gamma / \sqrt{t a_{t}}}\right)}{e^{\gamma / \sqrt{t a_{t}}}}\right)\right]
\end{aligned}
$$

and, by taking into account (14), one can easily check that

$$
a_{t} \log \left(F_{k}\left(e^{\gamma / \sqrt{t a_{t}}}, t\right) e^{-\gamma \mathbb{E}_{k}[N(t)] / \sqrt{t a_{t}}}\right)=A_{1}(t)+A_{2}(t)
$$


where

$$
A_{1}(t):=t a_{t}\left(\Lambda\left(\frac{\gamma}{\sqrt{t a_{t}}}\right)+\frac{\gamma}{\sqrt{t a_{t}}} \frac{k-\mathbb{E}_{k}[N(t)]}{t}\right)
$$

and

$$
A_{2}(t):=t a_{t}\left(\frac{1}{t} \log \left[\cosh \left(t \frac{h\left(e^{\gamma / \sqrt{t a_{t}}}\right)}{e^{\gamma / \sqrt{t a_{t}}}}\right)+\frac{c_{k}\left(e^{\gamma / \sqrt{t a_{t}}}\right)}{h\left(e^{\gamma / \sqrt{t a_{t}}}\right)} \sinh \left(t \frac{h\left(e^{\gamma / \sqrt{t a_{t}}}\right)}{e^{\gamma / \sqrt{t a_{t}}}}\right)\right]-\frac{h\left(e^{\gamma / \sqrt{t a_{t}}}\right)}{e^{\gamma / \sqrt{t a_{t}}}}\right) .
$$

Then we complete the proof by taking the limits of $A_{1}(t)$ and $A_{2}(t)$ as $t \rightarrow+\infty$. Firstly, by (12) and (18) (here we consider the Taylor Formula for $\Lambda\left(\frac{\gamma}{\sqrt{t a_{t}}}\right)$ ), we get

$$
\begin{aligned}
\lim _{t \rightarrow+\infty} A_{1}(t)=\lim _{t \rightarrow+\infty} t a_{t} & \left(\Lambda(0)+\Lambda^{\prime}(0) \frac{\gamma}{\sqrt{t a_{t}}}+\frac{1}{2} \Lambda^{\prime \prime}(0) \frac{\gamma^{2}}{t a_{t}}+o\left(\frac{\gamma^{2}}{t a_{t}}\right)\right. \\
& \left.+\frac{\gamma}{\sqrt{t a_{t}}}\left(-\frac{m_{k}\left(1-e^{-2(\lambda+\mu) t}\right)}{t}-\Lambda^{\prime}(0)\right)\right)=\frac{1}{2} \Lambda^{\prime \prime}(0) \gamma^{2} .
\end{aligned}
$$

Moreover we have $\lim _{t \rightarrow+\infty} A_{2}(t)=0$ by noting that there exists $M>0$ (which does not depend on $t$ ) such that

$$
\frac{1}{2} e^{t \frac{h\left(e^{\gamma / \sqrt{t a_{t}}}\right)}{e^{\gamma / \sqrt{t a_{t}}}}} \leq \cosh \left(t \frac{h\left(e^{\gamma / \sqrt{t a_{t}}}\right)}{e^{\gamma / \sqrt{t a_{t}}}}\right)+\frac{c_{k}\left(e^{\gamma / \sqrt{t a_{t}}}\right)}{h\left(e^{\gamma / \sqrt{t a_{t}}}\right)} \sinh \left(t \frac{h\left(e^{\gamma / \sqrt{t a_{t}}}\right)}{e^{\gamma / \sqrt{t a_{t}}}}\right) \leq(1+M) e^{t \frac{h\left(e^{\gamma / \sqrt{t a_{t}}}\right)}{e^{\gamma / \sqrt{t a_{t}}}}}
$$

for all $t$ large enough (actually the lower bound holds for all $t>0$ ). Thus (21) is satisfied and the proof is complete.

Finally, due to Remark 2.2 we note that

$$
\Lambda^{\prime \prime}(0)=\lim _{t \rightarrow+\infty} \frac{\operatorname{Var}_{k}[N(t)]}{t}
$$

for all $k \in \mathbb{Z}$. Thus, as typically happens for the LDPs in the regime of moderate deviations, $\Lambda^{\prime \prime}(0)$ can be considered as an asymptotic variance.

\subsection{A lower bound for level crossing probabilities}

In various papers such as [Stockmayer et al. (1971)] heterogeneous chains of two regularly alternating kinds of atoms are considered, in which the atoms are joined by links that are exposed to random shocks. Such shocks cause atoms to move in the chain and the molecule to diffuse, so that the position an atom occupies within the chain at time $t$ is well described by the process $N(t)$. In this context it is relevant to study level crossing problems in order to assess information on the reachability of given positions of the chain. Hence, motivated by possible applications in chemical physics, now we prove an asymptotic lower bound for the level crossing probabilities

$$
\Psi_{N}(u, T):=P(\{\exists t \in[0, T], N(t)>u\} \mid N(0)=0)
$$


and

$$
\Psi_{N}(u):=P(\{\exists t \in[0,+\infty), N(t)>u\} \mid N(0)=0) .
$$

We remark that $\Psi_{N}(u)$ goes to zero as $u \rightarrow+\infty$ if and only if $\Lambda^{\prime}(0)<0$, and we can refer to the discussion in Remark 3.1.

Some preliminaries are needed. We define

$$
w:=\inf \{\gamma \in \mathbb{R}: \Lambda(\gamma) \leq 0\}
$$

We remark that $w \geq 0$ and $\Lambda^{\prime}(w) \geq 0$. Note that $\Lambda^{\prime}(w)=0$ only if $w=0$. There exist two cases: $w=0$ if $\Lambda^{\prime}(0) \geq 0$, and $w>0$ if $\Lambda^{\prime}(0)<0$. In the second case $w$ is the unique positive value such that $\Lambda(w)=0$. We also introduce the function $\rho(\cdot)$ defined by

$$
\rho(T):= \begin{cases}T \Lambda^{*}(1 / T) & \text { if } T \in\left(0,1 / \Lambda^{\prime}(w)\right) \\ w & \text { if } T \in\left[1 / \Lambda^{\prime}(w),+\infty\right)\end{cases}
$$

for $T>0$; note that the case $T \geq 1 / \Lambda^{\prime}(w)$ is empty when $\Lambda^{\prime}(w)=0$.

The following result is related to Proposition 3.1.

Corollary 3.3 The following inequalities hold:

(i) $\liminf \operatorname{in}_{u \rightarrow+\infty} \frac{1}{u} \log \Psi_{N}(u, u T) \geq-\rho(T)$;

(ii) $\liminf \inf _{u \rightarrow+\infty} \frac{1}{u} \log \Psi_{N}(u) \geq-w$.

Proof. For $s \in(0, T]$ we have

$$
\begin{aligned}
\liminf _{u \rightarrow+\infty} \frac{1}{u} \log \Psi_{N}(u, u T) & \geq s \liminf _{u \rightarrow+\infty} \frac{1}{u s} \log P\left(\frac{N(u s)}{u s}>\frac{1}{s} \mid N(0)=0\right) \\
& \geq-s \inf \left\{\Lambda^{*}(z): z>1 / s\right\}
\end{aligned}
$$

by the inequality $\Psi_{N}(u, u T) \geq P(N(u s)>u \mid N(0)=0)$ and by the LDP lower bound in Proposition 3.1. Thus we only have to check that

$$
\rho(T)=\inf \left\{s \inf \left\{\Lambda^{*}(z): z>1 / s\right\}: s \in(0, T]\right\} .
$$

This can be easily verified when $\Lambda^{\prime}(0) \geq 0$ and $T \geq \frac{1}{\Lambda^{\prime}(w)}$ because $w=0$, and therefore $\inf \left\{\Lambda^{*}(z)\right.$ : $z>1 / T\}=0$. In all other cases we have to check that

$$
\rho(T)=\inf \left\{s \Lambda^{*}(1 / s): s \in(0, T]\right\}
$$

by the continuity of $\Lambda^{*}$. In view of what follows we consider the function $s \mapsto \gamma_{s}$ on $(0,+\infty)$, where $\gamma_{s}$ is the unique value such that $\Lambda^{\prime}\left(\gamma_{s}\right)=1 / s$. This function is well-defined by the strict convexity of $\Lambda$; moreover it is a regular function (indeed we have $\gamma_{s}^{\prime}=\left(\Lambda^{*}\right)^{\prime}(1 / s)$ by a known argument of convex analysis, see e.g. Theorem 26.5 in [Rockafellar (1970)]). Then we obtain

$$
s \Lambda^{*}(1 / s)=s\left\{\frac{\gamma_{s}}{s}-\Lambda\left(\gamma_{s}\right)\right\}=\gamma_{s}-s \Lambda\left(\gamma_{s}\right),
$$


so that

$$
\frac{d}{d s} s \Lambda^{*}(1 / s)=\frac{d}{d s}\left\{\gamma_{s}-s \Lambda\left(\gamma_{s}\right)\right\}=\gamma_{s}^{\prime}-\Lambda\left(\gamma_{s}\right)-s \Lambda^{\prime}\left(\gamma_{s}\right) \gamma_{s}^{\prime}=-\Lambda\left(\gamma_{s}\right) .
$$

In conclusion one can check the equality (22) in the following three cases:

$$
\begin{array}{lll}
\frac{d}{d s} s \Lambda^{*}(1 / s)=0, & \gamma_{s}=w, & s=1 / \Lambda^{\prime}(w) ; \\
\frac{d}{d s} s \Lambda^{*}(1 / s)>0, & \gamma_{s} \in(0, w), & s \in\left(1 / \Lambda^{\prime}(w),+\infty\right) ; \\
\frac{d}{d s} s \Lambda^{*}(1 / s)<0, & \gamma_{s} \in(w,+\infty), & s \in\left(0,1 / \Lambda^{\prime}(w)\right) .
\end{array}
$$

This completes the proof of (i). Moreover, for all $T>0$ we have

$$
\liminf _{u \rightarrow+\infty} \frac{1}{u} \log \Psi_{N}(u) \geq \liminf _{u \rightarrow+\infty} \frac{1}{u} \log \Psi_{N}(u, u T) \geq-\rho(T)
$$

by the inequality $\Psi_{N}(u) \geq \Psi_{N}(u, u T)$ and by point (i) of this proposition. Hence,

$$
\liminf _{u \rightarrow+\infty} \frac{1}{u} \log \Psi_{N}(u) \geq-\inf \{\rho(T): T>0\}=-\lim _{T \rightarrow+\infty} \rho(T)=-w
$$

because $\rho(\cdot)$ is a decreasing function. The proof of (ii) is thus completed.

\section{Appendix}

The statement of the Gärtner Ellis Theorem. For the sake of completeness we recall here the statement of the Gärtner Ellis Theorem; here we present the result by referring to Theorem 2.3.6(c) in [Dembo and Zeitouni (1998)] for a family of probability measures (instead of random variables) and a speed function $v_{t}$. Moreover we restrict the attention to the one dimensional case $(d=1)$, and we also refer to Definition 2.3.5 in [Dembo and Zeitouni (1998)] for the concept of essentially smooth function.

Let $\left\{\pi_{t}: t>0\right\}$ be a family of probability measures on $\mathbb{R}$ such that, for all $\gamma \in \mathbb{R}$, the limit

$$
\Lambda(\gamma):=\lim _{t \rightarrow+\infty} \frac{1}{v_{t}} \log \int_{\mathbb{R}} e^{v_{t} \gamma x} \pi_{t}(d x)
$$

exists as an extended real number, and consider the set $\mathcal{D}_{\Lambda}:=\{\gamma \in \mathbb{R}: \Lambda(\gamma)<\infty\}$. Then, if the function $\Lambda$ is essentially smooth and lower semi-continuous, $\left\{\pi_{t}: t>0\right\}$ satisfies the rate function with speed function $v_{t}$ and good rate function $\Lambda^{*}$ defined by $\Lambda^{*}(x):=\sup _{\gamma \in \mathbb{R}}\{\gamma x-\Lambda(\gamma)\}$.

We recall that the convex function $\Lambda$ is essentially smooth if: the interior $\mathcal{D}_{\Lambda}^{\circ}$ of $\mathcal{D}_{\Lambda}$ is nonempty, the function $\Lambda$ is differentiable throughout $\mathcal{D}_{\Lambda}^{\circ}$, and $\left|\Lambda^{\prime}\left(\gamma_{n}\right)\right|$ goes to infinity whenever $\left\{\gamma_{n}: n \geq 1\right\}$ is a sequence of points in $\mathcal{D}_{\Lambda}^{\circ}$ converging to a boundary point of $\mathcal{D}_{\Lambda}^{\circ}$ as $n \rightarrow+\infty$. We remark that the last condition trivially holds when $\mathcal{D}_{\Lambda}=\mathbb{R}$; in fact in this case the boundary of $\mathcal{D}_{\Lambda}^{\circ}$ is empty. 
The proof of (19). Firstly by (3), (5), (12) and (18) we have

$$
\begin{aligned}
\log \mathbb{E}_{k}\left[e^{\left.\gamma \frac{N(t)-\mathbb{E}_{k}[N(t)]}{\sqrt{t}}\right]=}\right. & \log \left(F_{k}\left(e^{\gamma / \sqrt{t}}, t\right) e^{-\gamma \mathbb{E}_{k}[N(t)] / \sqrt{t}}\right) \\
= & -\frac{\gamma}{\sqrt{t}} m_{k}\left(1-e^{-2(\lambda+\mu) t}\right)+t\{-(\lambda+\mu) \\
& \left.+\frac{1}{t} \log \left[\cosh \left(t \frac{h\left(e^{\gamma / \sqrt{t}}\right)}{e^{\gamma / \sqrt{t}}}\right)+\frac{c_{k}\left(e^{\gamma / \sqrt{t}}\right)}{h\left(e^{\gamma / \sqrt{t}}\right)} \sinh \left(t \frac{h\left(e^{\gamma / \sqrt{t}}\right)}{e^{\gamma / \sqrt{t}}}\right)\right]-\frac{\gamma}{\sqrt{t}} \Lambda^{\prime}(0)\right\} .
\end{aligned}
$$

Thus, if we set

$$
A_{1}(t):=\Lambda\left(\frac{\gamma}{\sqrt{t}}\right)-\frac{\gamma}{\sqrt{t}} \Lambda^{\prime}(0)=\frac{h\left(e^{\gamma / \sqrt{t}}\right)}{e^{\gamma / \sqrt{t}}}-(\lambda+\mu)-\frac{\gamma}{\sqrt{t}} \Lambda^{\prime}(0)
$$

and

$$
A_{2}(t):=\frac{1}{t} \log \left[\cosh \left(t \frac{h\left(e^{\gamma / \sqrt{t}}\right)}{e^{\gamma / \sqrt{t}}}\right)+\frac{c_{k}\left(e^{\gamma / \sqrt{t}}\right)}{h\left(e^{\gamma / \sqrt{t}}\right)} \sinh \left(t \frac{h\left(e^{\gamma / \sqrt{t}}\right)}{e^{\gamma / \sqrt{t}}}\right)\right]-\frac{h\left(e^{\gamma / \sqrt{t}}\right)}{e^{\gamma / \sqrt{t}}}
$$

we obtain

$$
\log \mathbb{E}_{k}\left[e^{\gamma \frac{N(t)-\mathbb{E}_{k}[N(t)]}{\sqrt{t}}}\right]=-\frac{\gamma}{\sqrt{t}} m_{k}\left(1-e^{-2(\lambda+\mu) t}\right)+t\left\{A_{1}(t)+A_{2}(t)\right\} .
$$

Finally we get (19) by noting that

$$
\begin{gathered}
\lim _{t \rightarrow+\infty} \frac{\gamma}{\sqrt{t}} m_{k}\left(1-e^{-2(\lambda+\mu) t}\right)=0 \\
\lim _{t \rightarrow+\infty} t A_{1}(t)=\lim _{t \rightarrow+\infty} t\left\{\Lambda(0)+\frac{\gamma}{\sqrt{t}} \Lambda^{\prime}(0)+\frac{1}{2} \Lambda^{\prime \prime}(0) \frac{\gamma^{2}}{t}+o\left(\frac{\gamma^{2}}{t}\right)-\frac{\gamma}{\sqrt{t}} \Lambda^{\prime}(0)\right\}=\frac{1}{2} \Lambda^{\prime \prime}(0) \gamma^{2},
\end{gathered}
$$

where we consider the Taylor Formula for $\Lambda\left(\frac{\gamma}{\sqrt{t}}\right)$, and

$$
\lim _{t \rightarrow+\infty} t A_{2}(t)=\lim _{t \rightarrow+\infty} \log \left(\frac{\cosh \left(t \frac{h\left(e^{\gamma / \sqrt{t}}\right)}{e^{\gamma / \sqrt{t}}}\right)+\frac{c_{k}\left(e^{\gamma / \sqrt{t}}\right)}{h\left(e^{\gamma / \sqrt{t}}\right)} \sinh \left(t \frac{h\left(e^{\gamma / \sqrt{t}}\right)}{e^{\gamma / \sqrt{t}}}\right)}{e^{t \frac{h\left(e^{\gamma / \sqrt{t}}\right)}{e^{\gamma / \sqrt{t}}}}}\right)=\log \left(\frac{1}{2}+\frac{1}{2}\right)=0 .
$$

\section{Acknowledgements}

The authors thank the two anonymous referees for helpful comments that improved the paper.

\section{References}

[Barrera et al. (2009)] Barrera, J., Bertoncini, O., Fernández, R., 2009. Abrupt convergence and escape behavior for birth and death chains. J. Stat. Phys. 137, 595-623.

[Böhm and Hornik (2010)] Böhm, W., Hornik, K., 2010. On two-periodic random walks with boundaries. Stoch. Models 26, 165-194. 
[Bryc (1993)] Bryc, W., 1993. A remark on the connection between the large deviation principle and the central limit theorem. Stat. Prob. Lett. 18, 253-256.

[Chan (1998)] Chan, T., 1998. Large deviations and quasi-stationarity for density-dependent birth-death processes. J. Austral. Math. Soc. Ser. B 40, 238-256.

[Conolly (1971)] Conolly, B.W., 1971. On randomized random walks. SIAM Review 13, 81-99.

[Conolly et al. (1997)] Conolly, B.W., Parthasarathy, P.R., Dharmaraja, S., 1997. A chemical queue. Math. Scientist 22, 83-91.

[Cox and Griffeath (1984)] Cox, J.T., Griffeath, D., 1984. Large deviations for Poisson systems of independent random walks. Z. Wahrsch. Verw. Gebiete 66, 543-558.

[de Acosta (1994)] de Acosta, A., 1994. Large deviations for vector valued Lévy processes. Stochastic Process. Appl. 51, 75-115.

[Dembo and Zeitouni (1998)] Dembo, A., Zeitouni, O., 1998. Large Deviations Techniques and Applications. 2nd Edition, Springer.

[Di Crescenzo et al. (2012)] Di Crescenzo, A., Iuliano, A., Martinucci, B., 2012. On a bilateral birth-death process with alternating rates. Ricerche Mat. 61, 157-169.

[Ermolaev and Külske (2010)] Ermolaev, V., Külske, C., 2010. Low-temperature dynamics of the Curie-Weiss model: periodic orbits, multiple histories, and loss of gibbsianness. J. Stat. Phys. $141,727-756$.

[Feng and Kurtz (2006)] Feng, J., Kurtz, T.G., 2006. Large Deviations for Stochastic Processes. American Mathematical Society.

[Pakdaman et al. (2010)] Pakdaman, K., Thieullen, M., Wainrib, G., 2010. Diffusion approximation of birth-death processes: comparison in terms of large deviations and exit points. Stat. Prob. Lett. 80, 1121-1127.

[Parthasarathy and Dharmaraja (1998)] Parthasarathy, P.R., Dharmaraja, S., 1998. The transient solution of a local-jump heterogeneous chain of diatomic systems. J. Phys. A: Math. Gen. 31, 6579-6588.

[Pruitt (1963)] Pruitt, W.E., 1963. Bilateral birth and death processes. Trans. Amer. Math. Soc. $107,508-525$.

[Rockafellar (1970)] Rockafellar, R.T., 1970. Convex Analysis. Princeton University Press. 
[Redig and Wang (2012)] Redig, F., Wang, F., 2012. Gibbs-Non-Gibbs transitions via large deviations: computable examples. J. Stat. Phys. 147, 1094-1112.

[Shwartz and Weiss (1995)] Shwartz, A., Weiss, A., 1995. Large Deviations for Performance Analysis. Chapman Hall.

[Stockmayer et al. (1971)] Stockmayer, W.H., Gobush, W., Norvich, R., 1971. Local-jump models for chain dynamics. Pure Appl. Chem. 26, 555-561.

[Tarabia (2009)] Tarabia, A.M.K., 2009. Analysis of random walks with an absorbing barrier and chemical rule. J. Comput. Appl. Math. 225, 612-620.

[Tarabia and El-Baz (2007)] Tarabia, A.M.K., El-Baz, A.H., 2007. Transient solution of a random walk with chemical rule. Physica A 382, 430-438.

[Tarabia et al. (2009)] Tarabia, A.M.K., Takagi H., El-Baz, A.H., 2009. Transient solution of a non-empty chemical queueing system Math. Meth. Oper. Res. 70, 77-98. 